\title{
BARYONS: EXTRACTING INFORMATION ON STRUCTURE FROM DATA*
}

\author{
M. T. PEÑA \\ Centro de Física Teórica de Partículas, CFTP, \\ Instituto Superior Técnico, Universidade de Lisboa, \\ Avenida Rovisco Pais, 1049-001, Lisboa, Portugal \\ teresa.pena@ist.utl.pt
}

Published 14 January 2014

\begin{abstract}
A new era of physical insight and understanding on the $\mathrm{N}^{*} \mathrm{~s}$ and their electrocouplings became possible by electron beam facilities and large acceptance detectors, as for example, at JLab. We address issues such as how the new experimental results elucidate us on the strong interaction dynamics in the non-perturbative regime, and what is the possible role of models, and their connection to LQCD data.
\end{abstract}

\section{Introduction}

Baryons were at the root of the development of the quark model, and constituent quark models ${ }^{1}$ are one of the theoretical paths to the understanding of the structure of those systems. There are other theoretical approaches, from Coupled-Channel Dynamical Models and Chiral Perturbation Theory, to perturbative QCD, QCD in the large $N_{c}$ limit, QCD simulations in the lattice, and Dyson-Schwinger formulations. All these approaches are represented and debated in the program of this meeting. One conclusion from comparing views and calculational schemes underlying these different approaches is that sometimes they have to be connected for mutual benefit and more insight. To illustrate this point, I will connect a constituent quark model to LQCD data. In doing so we gain information that would not be directly accessed by any of the two approaches separately.

\section{Covariant Spectator Theory Model}

I will focus on the so-called Covariant Spectator Theory (CST), a field theoretic based framework, and its applications to Hadron Physics. The results will show

\footnotetext{
*This is an Open Access article published by World Scientific Publishing Company. It is distributed under the terms of the Creative Commons Attribution 3.0 (CC-BY) License. Further distribution of this work is permitted, provided the original work is properly cited.
} 
that the CST approach enables us to describe the behavior of several $\gamma N \rightarrow N^{*}$ hadronic vertices in an efficient way, at least in the high $Q^{2}$ region. In our covariant constituent quark model based on the CST, the quarks are dressed and have effective masses, anomalous magnetic moments, and effective electromagnetic form factors. Our starting assumption is that for reasonably high momentum transfer the coupling of a baryon with a photon can be calculated in impulse approximation. This means that the photon couples with only one of the three constituent quarks at a time, as illustrated in Fig.1. To build the model we calculate this diagram, and we proceed through a sequence of three steps:

1) Within CST the covariant 4D integration over the momenta of the two non-active (meaning not interacting with the photon) quarks is reduced to a $3 \mathrm{D}$ integration by, in the energy integration, selecting the positive energy poles of those two quarks (which we call here the diquark);

2) One makes an intermediate variable transformation from the individual threemomenta $\mathbf{k}_{\mathbf{1}}$ and $\mathbf{k}_{\mathbf{2}}$ of the two quarks in the diquark, to the (Jacobi) variables of the relative quark-diquark motion $\mathbf{k}$ and the internal relative momentum $\mathbf{r}$ of the diquark pair, followed by another transformation from $\mathbf{r}$ to the diquark invariant mass $s$ and the orientation $\Omega_{\hat{\mathbf{r}}}$ of its internal relative momentum $\mathbf{r}$;

3) Since the electromagnetic coupling does not depend on $s$ or $\Omega_{\hat{\mathbf{r}}}$, we can integrate over those variables — which leaves us with only the integration over the quarkdiquark relative momentum $k$ involving the active off-mass-shell quark that interacts with the photon.

These three steps can be mathematically summarized by the sequence

$$
\int d^{4} k_{1} d^{4} k_{2} \rightarrow \int \frac{d^{3} k_{1}}{E_{k_{1}}} \frac{d^{3} k_{2}}{E_{k_{2}}} \rightarrow \underbrace{\int \frac{d \Omega_{\hat{\mathbf{r}}}}{4(2 \pi)^{3}} \int_{4 m_{q}^{2}}^{\infty} d s \sqrt{\frac{s-4 m_{q}^{2}}{s}}}_{\int_{s}} \underbrace{\int \frac{d^{3} k}{(2 \pi)^{3} 2 E_{s}}}_{\int_{k}} \equiv \int_{s k},
$$

where the first step represents the CST prescription for the energy integration, and $m_{q}$ is the constituent quark mass. The conclusion to be drawn at this point is that within impulse approximation it suffices to start with an effective quark-diquark structure for the baryon vertex. This vertex is to be interpreted as resulting from an integration of the three-body vertex over the diquark internal variables. Although the true three-quark structure of the baryon is certainly decisive for a description

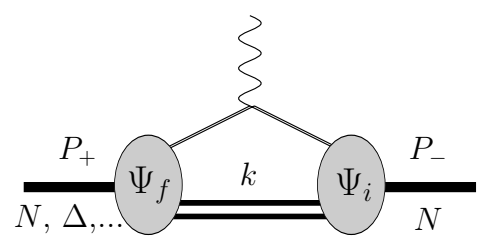

Fig. 1. Diagram for the coupling of the baryons with a photon within impulse approximation. 
of the baryon spectra, it follows from the steps above that the microscopic 3-body structure may not be as important for the electromagnetic form factors, at least in the energy regime where the impulse approximation is valid.

If one labels the off-mass-shell quark interacting with the photon as quark 3 , the electromagnetic transition current in relativistic impulse approximation is given by the diagram of Fig. 1 and reads ${ }^{2,6,5}$

$$
J_{N R}^{\mu}=3 \sum_{\Gamma} \int_{k} \bar{\Psi}_{f}\left(P_{+}, k\right) j_{q}^{\mu} \Psi_{i}\left(P_{-}, k\right),
$$

where $j_{q}^{\mu}$ is the quark current associated with constituent quark 3, and the sum is over the diquark spin states $\Gamma$. These states include a spin 1 vector component with polarization $\Lambda=0, \pm$. For baryons of spin $1 / 2$ as the nucleon, the sum includes also a scalar spin 0 diquark component. ${ }^{2,3}$ The factor 3 takes into account the contributions of the other two possible interacting quarks/diquark pairs, as demanded by symmetry. The initial and final baryon effective quark-diquark wave functions $\Psi_{i}\left(P_{-}, k\right)$ and $\Psi_{f}\left(P_{+}, k\right)$ have a general form that satisfies the correct symmetries in the spin-flavor and momentum space, include radial and orbital motion, and are written in terms of the diquark polarization vector states, $\varepsilon_{\Lambda P}^{\alpha{ }^{2,3}}$

Because the quarks are effectively dressed, the single constituent quark current $j_{q}^{\mu}$ in equation (2) decomposes into two terms

$$
j_{q}^{\mu}=j_{1} \hat{\gamma}^{\mu}+j_{2} \frac{i \sigma^{\mu \nu} q_{\nu}}{2 M}, \hat{\gamma}^{\mu}=\gamma^{\mu}-\frac{\not q q^{\mu}}{q^{2}},
$$

where $M$ is the nucleon mass, $j_{1}$ and $j_{2}$ are the Dirac and Pauli quark form factors. Each of these form factors $j_{i}(i=1,2)$ have an isoscalar and an isovector component, given respectively by the functions $f_{i+}$ and $f_{i-}$ (of $Q^{2}$, the 4-momentum transfer squared), as $j_{i}=\frac{1}{6} f_{i+}+\frac{1}{2} f_{i-} \tau_{3}$. The explicit forms of the Dirac and Pauli quark form factors, $f_{1 \pm}$ and $f_{2 \pm}$ respectively, are chosen to be consistent with the vector meson dominance (VMD) mechanism, being parametrized as ${ }^{2,3}$

$$
\begin{aligned}
& f_{1 \pm}\left(Q^{2}\right)=\lambda_{q}+\left(1-\lambda_{q}\right) \frac{m_{v}^{2}}{m_{v}^{2}+Q^{2}}+c_{ \pm} \frac{M_{h}^{2} Q^{2}}{\left(M_{h}^{2}+Q^{2}\right)^{2}} \\
& f_{2 \pm}\left(Q^{2}\right)=\kappa_{ \pm}\left\{d_{ \pm} \frac{m_{v}^{2}}{m_{v}^{2}+Q^{2}}+\left(1-d_{ \pm}\right) \frac{M_{h}^{2}}{M_{h}^{2}+Q^{2}}\right\}
\end{aligned}
$$

where $m_{v}$ is a light vector meson mass, $M_{h}$ is a mass of an effective heavy vector meson, $\kappa_{ \pm}$are quark anomalous magnetic moments. The mixture coefficients $c_{ \pm}, d_{ \pm}$ are phenomenologicaly fixed by the proton and neutron elastic electromagnetic form factors. The parameter $\lambda_{q}$ is related to the quark density number and fixed by deep inelastic scattering data. In the applications we took $m_{v}=m_{\rho}\left(\simeq m_{\omega}\right)$ to include the physics associated with the $\rho$-pole and $M_{h}=2 M$ (twice the nucleon mass) to take into account effects of meson resonances with a larger mass. The quark form factors are moreover normalized to reproduce the charge and anomalous magnetic moment of the $u$ and $d$ quarks. 
Finally, the spin part of the wave functions for several baryon systems ${ }^{2-6}$ are written in terms of the states $U_{R}^{\alpha}(P, s)=\frac{1}{\sqrt{3}} \gamma_{5}\left(\gamma^{\alpha}-\frac{P^{\alpha}}{M_{R}}\right) u_{R}(P, s)$, where $u_{R}$ is the Dirac spinor for any particle $R$, for core spin $1 / 2$ state components, and in terms of the Rarita-Schwinger state $u_{\alpha}$, for core spin $3 / 2$ state components. It was then possible to construct for several baryons wave functions that are explicitly covariant, have the correct nonrelativistic limit, ${ }^{2,3}$ and include in a pratical way components with angular momentum $L>0$, in particular $P$ and $D$ waves.

\section{Connection of the Model to LQCD}

The connection to LQCD arose from the following realizations: ${ }^{7,8} 1$ ) the pion cloud effects are negligible for large pion masses, 2) our bare quark core model can be calibrated by the LQCD data for large pion masses, since it uses an electromagnetic quark current inspired by the mechanism of vector meson dominance, and the vector meson mass can be written as a function of the running pion mass, 3) after that, by taking the limit of the model back to the physical pion mass value, the experimental data is well described, at least in the high momentum transfer $Q^{2}$ region.

It was in the $N \gamma \rightarrow \Delta(1232)$ excitation that this connection was first checked in practice. ${ }^{7,8}$ We fixed the $\Delta(1232)$ wave function by calibrating it to the LQCD results for the $N \gamma \rightarrow \Delta(1232)$ form factors, and this calibration made use of a running pion mass to calculate the $\rho$ meson mass. Afterwards, by restoring the model to the physical pion mass limit, we could successfully predict the behavior of the form factors in the large $Q^{2}$ region. In addition, we made the assumption that for all the three form factors of the reaction the contributions from the constituent quark core and from the pion cloud can be added, with no interference effects. This is supported by the experimental data for the dominant form factor, $G_{M}{ }^{3}$ as pointed out in other talks during this meeting. Then, by subtracting the experimental data from our constituent quark model, we could make estimations for the pion cloud effects, which were non-zero in the vicinity of $Q^{2} \approx 0$. Three important conclusions of this work were: 1 ) the extracted information on the pion cloud contribution to the $\Delta(1232)$ excitation is consistent with the EBAC analysis ${ }^{9}$ and also with the results from QCD in the large $N_{c}$ limit, ${ }^{10} 2$ ) by doing first the fit of the form factors to the LQCD data one can predict the experimental data in the regime of the physical pion mass, however, the reverse is not true: by fitting the physical data one is not successful in describing the LQCD data, 3) the weight of the $D$ wave component in the $\Delta(1232)$ wave function could only be well constrained by the LQCD data, and in contrast, the experimental data alone does not fix it at a reasonable value.

\section{Results}

Besides the reaction for the excitation of the nucleon to the $\Delta(1232),{ }^{4}$ applications of our model include the excitation reactions of the $N^{*}(1440),{ }^{11} N^{*}(1535),{ }^{12}$ $\Delta(1600),{ }^{13}$ the baryon octet, ${ }^{14}$ and more recently, the $N^{*}(1520)$. Other applications 
are the form factors of the $\Delta^{15}$ and of the $\Omega^{-}$baryon, ${ }^{5}$ Deep Inelastic Scattering, ${ }^{6}$ the dilepton mass spectrum, ${ }^{16}$ and effects of the nuclear medium. ${ }^{17}$ Many of these applications are predictions of our model, without further parameter fitting, once the form factors of the nucleon and of the $N \gamma \rightarrow N^{*}(1232)$ transition were described.

Finally, we briefly present very recent preliminary results from our work on the $N \gamma \rightarrow N^{*}(1520)$ transition, still in progress. The $N^{*}(1520)$ has spin $3 / 2$, and negative parity, and has an important role in dielectric production processes at intermediate energies. Figs. 2 and 3 show the results for the helicity amplitudes, $A_{1 / 2}, S_{1 / 2}$, and $A_{3 / 2}$ describing this reaction. In the left panel of Fig. 2 the dashed line is close to the data in the large $Q^{2}$ region, showing that the two baryons, $N$ and $N^{*}(1520)$, have a very similar structure in their inner core, probed in the high momentum transfer region. This was also seen for the $N^{*}(1535) .{ }^{12}$ The $A_{3 / 2}$ helicity amplitude (Fig. 2) is found to be negligible in our quark core model — it originates only from the coupling of an orbital $P$ wave to a core spin state $S=3 / 2$, which is dynamically suppressed compared to the core spin $S=1 / 2$ state. Similarly to other $N^{*}$ s, our model provides information on the pion cloud effects for all the helicity amplitudes of this reaction, as shown in the right panel of Fig. 2 and in Fig. 3. It
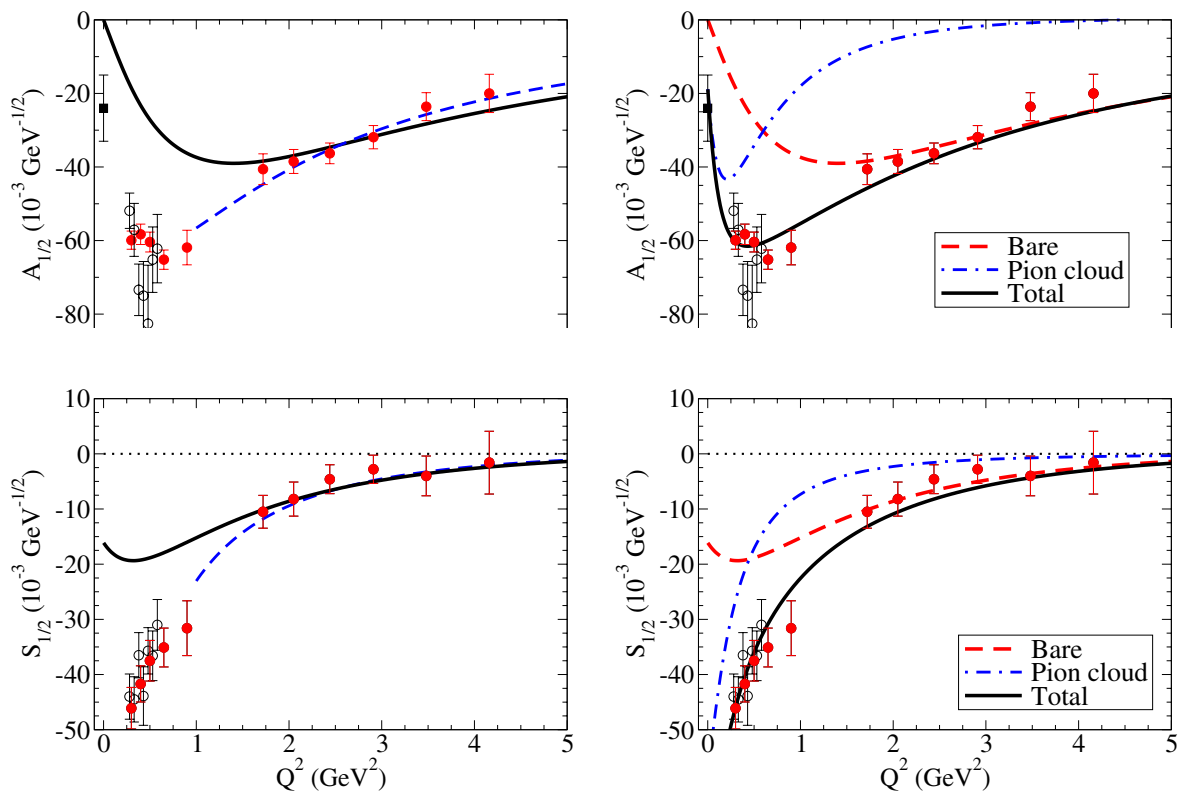

Fig. 2. Left: Valence quark contributions for two of the three helicity amplitudes describing the excitation of the $\mathrm{N}^{*}(1520)$. The dashed line corresponds to a wave function for the $\mathrm{N}^{*}(1525)$ that is the same as for the nucleon. The solid line is the result from a model imposing orthogonality between these two baryon wave functions, and determined by a fit to the data for $Q^{2}>1.5 \mathrm{GeV}^{2}$. Right: Valence quark and pion cloud contributions for the same amplitudes. Data from Refs. 18 (full circles) and 19 (empty circles). 


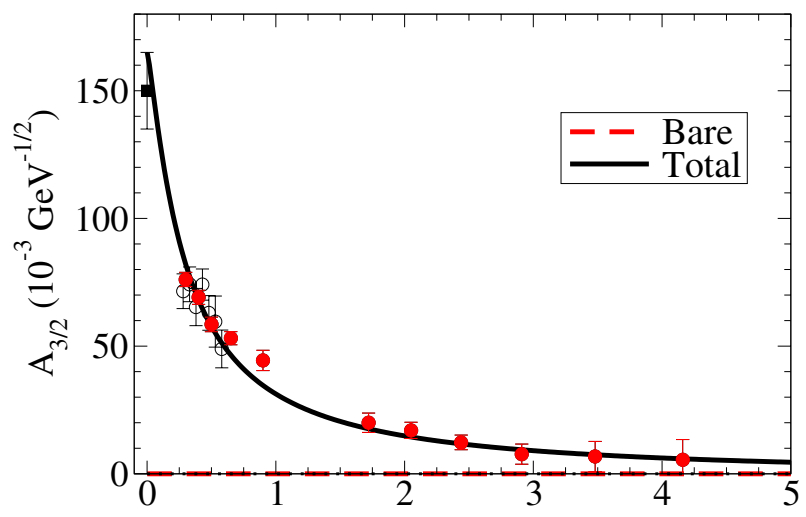

Fig. 3. Pion cloud contribution for the $A_{3 / 2}$ amplitude of the excitation of the $\mathrm{N}^{*}(1520)$. The pion cloud effect practically coincides with the total result. Data as in Fig. 2.

also describes the data for high $Q^{2}$ where our results confirm the dominance of the $A_{1 / 2}$ amplitude. ${ }^{20}$

\section{Acknowledgments}

This work was supported by FCT - Fundação para a Ciência e a Tecnologia, Grants PEst-OE/FIS/UI0777/2011 and PTDC/FIS/113940/2009, and the EU, HadronPhysics3 Grant No. 283286. I thank my collaborators G. Ramalho and Franz Gross.

\section{References}

1. F. E. Close and F. J. Gilman, Phys. Lett. B 38, 541 (1972); M. Aiello, M. M. Giannini and E. Santopinto, J. Phys. G 24, 753 (1998).

2. F. Gross, G. Ramalho and M. T. Peña, Phys. Rev. C 77, 015202 (2008).

3. G. Ramalho, M. T. Peña and F. Gross, Eur. Phys. J. A 36, 329 (2008).

4. G. Ramalho, M. T. Peña and F. Gross, Phys. Rev. D 78, 114017 (2008).

5. G. Ramalho and M. T. Peña, Phys. Rev. D 83, 054011 (2011).

6. F. Gross, G. Ramalho and M. T. Peña, Phys. Rev. D 85, 093005 (2012)]; F. Gross, G. Ramalho and M. T. Peña, Phys. Rev. D 85, 093006 (2012).

7. G. Ramalho and M. T. Peña, Phys. Rev. D 80 (2009) 013008.

8. G. Ramalho and M. T. Peña, J. Phys. G 36, 115011 (2009).

9. B. Julia-Diaz, T. -S. H. Lee, A. Matsuyama, T. Sato and L. C. Smith, Phys. Rev. C 77, 045205 (2008).

10. V. Pascalutsa and M. Vanderhaeghen, Phys. Rev. D 76 111501(R) (2007).

11. G. Ramalho and K. Tsushima, Phys. Rev. D 81 (2010) 074020.

12. G. Ramalho and M. T. Peña, Phys. Rev. D 84, 033007 (2011).

13. G. Ramalho and K. Tsushima, Phys. Rev. D 82, 073007 (2010).

14. F. Gross, G. Ramalho and K. Tsushima, Phys. Lett. B 690, 183 (2010); G. Ramalho and K. Tsushima, Phys. Rev. D 84, 054014 (2011).

15. G. Ramalho, M. T. Peña and F. Gross, Phys. Rev. D 81, 113011 (2010);

16. G. Ramalho and M. T. Peña, Phys. Rev. D 85, 113014 (2012). 
17. G. Ramalho, K. Tsushima and A. W. Thomas, J. Phys. G 40, 015102 (2013).

18. I. G. Aznauryan et al. [CLAS Collaboration], Phys. Rev. C 80, 055203 (2009);

I. G. Aznauryan and V. D. Burkert, Prog. Part. Nucl. Phys. 67, 1 (2012).

19. V. I. Mokeev et al. [CLAS Collaboration], Phys. Rev. C 86, 035203 (2012).

20. I. G. Aznauryan and V. D. Burkert, Phys. Rev. C 85, 055202 (2012). 